\title{
Direct market access for exchange-traded derivatives
}

\author{
Frances Maguire \\ Email: fr@nces.fslife.co.uk
}

Received: 16th March, 2006

Frances Maguire is a freelance journalist with more than ten years' writing experience, specialising in topics such as derivatives, risk management, banking IT, securities processing and fund management. She was previously Associate Editor on Futures \& Options World, Editor of Futures \& Options Week, Banking Technology and technology writer for Financial News.

\begin{abstract}
Direct market access grew up out of the equities market in the late 1990s, in particular when the number of electronic marketplaces, or ECNs, for trading stocks greatly increased in the US, and spurred on again by the wider use of the FIX protocol for communicating indications of interest. Now that direct market access is fairly standard for stock trading, the same firms are looking to expand these operations to exchange-traded futures, made possible by the move to electronic trading by the derivatives exchanges.

While Europe and, to a certain extent, Asia-Pacific have grown up alongside electronic derivatives trading, with the London and Paris markets making the switch from open outcry trading to electronic fairly swiftly, the two biggest financial futures markets in Chicago have grown their electronic trading volumes, despite the fact that their open outcry

trading floors have remained open.

The speed at which the international exchange-traded derivatives markets have
\end{abstract} Derivatives Use, Trading \& Regulation, Vol. 11 No. 4, 2006 pp. $375-380$

(c) Palgrave Macmillan $1747-4426 / 06 \$ 30.00$ switched from open outcry trading to electronic trading has been spectacular. Volume on the Chicago Mercantile Exchange (CME) Globex electronics trading platform increased 62 per cent for the year, with total volume of 730 million contracts and average daily volume of 2.9 million. CME Globex volume represented 70 per cent of total CME volume, compared with 57 per cent in 2004.

The Chicago Board of Trade (CBOT) also experienced an increase in electronic trading volume, the latter representing 65 per cent of total exchange volume in 2005, up from 58 per cent in 2004. Furthermore, the electronic average daily volume grew 24 per cent to 1.7 million contracts from 1.4 million contracts reported in 2004, and the number of the CBOT's financial options that traded electronically surged by 180 per cent in 2005.

Electronic trading is a prerequisite for direct market access (DMA). It was only when the FIX protocol enabled fund 
managers to send electronic indications of interest that electronic communication of orders between firms truly became widespread. The growth of the number of equities marketplaces meant that DMA was essential to ensure fast and best execution.

\section{NEED FOR LIQUIDITY}

The second necessary prerequisite for DMA is liquidity. Where markets are illiquid, traders still reign. For this reason, DMA is only really used for exchange-traded futures contracts, and very rarely for options on futures traded at the same exchanges. The correlation between the growth of electronic trading and the growing use of DMA is clear in that only 10 per cent of options volume on CME is currently electronic, compared with around 80 per cent of the exchange's futures trading volume.

Simon Thompson, managing director and head of equity trading at Barclays Global Investors, oversees equities, equity derivatives trading and commodities, and says that BGI took its first steps to establish DMA to derivatives exchanges about a year ago. Besides the constant drive to harness optimal trading techniques, the two main reasons BGI is turning to DMA for derivatives is to gain control over trading and more importantly, gain complete anonymity. 'By going through a pipe, directly to the exchange, there is no trader at the other end at the broker. We are entering the orders directly onto the exchange. Where there is a large order, it is market sensitive and anonymity is a big issue', says Thompson.
The next step is the idea that buy-side can build their own algorithms to schedule trading strategies, for large orders, to operate in very fast, liquid markets. By crunching historic data and combining the profile of the order to create parameters and algorithms to conduct computerised trading, buy-side firms can build-in different levels of complexity for their own trading schedules. But for now, firms are using broker's algorithms, and for exchange-traded futures more than options.

He adds:

'Exchange-traded futures are well behind pure equities in terms of development.

At the moment, there are a limited number of brokers, which have a limited number of algorithms that they are making available to their clients. There are some hedge funds and quant shops that have developed their own algorithms for futures, but there are a limited number generically available to the buy-side.'

\section{BUY OR BUILD?}

Thompson adds, however, that while BGI is using DMA for derivatives, it does not necessarily mean there is a rush to using complex algorithms, similar to the equities markets. He says: 'We are not necessarily constantly building algorithms. FlexTrade gives us access to a broker electronically, and a broker can provide the algorithms. There is a lot we can buy from a broker before we start writing our own algorithms.'

Instead, FlexTrade provides GGI with a 
trading platform, FlexTrader, that takes real-time pricing input and indications of interest from brokers, via FIX and routes orders to multiple liquidity destinations exchanges, ECNs or brokers. This platform monitors $\mathrm{P} \& \mathrm{~L}$ in real time and is no different for equities and exchange-traded derivatives. Now that equities are well established on BGI's FlexTrader platform, the fund manager began accessing derivatives exchanges through FlexTrader earlier this year.

Other advantages of using DMA are the lower execution costs and the straight-through processing that computer-to-computer brings, enabling, according to Thompson, near elimination of errors. He says:

'Telephone orders can be misheard. If you are dealing direct from your own system, the communication of an order from a fund manager to your desk is electronic, you can only fire orders you physically have hold of, so there is no opportunity to over-trade or trade the wrong way due to the way that the front-end is set-up.'

Cost is a big driver towards DMA. By trading directly, DMA-execution costs are lower because a broker is not handling the execution.

Inevitably, the rise of DMA is beginning to affect exchange-traded derivatives. One upshot is the rise in the use of iceberg orders. The concept originated on the world's stock exchanges as a way of minimising the impact of the large buy or sell order from a single market participant.
Now, adopted by the derivatives exchanges, iceberg orders mean that a buy-side firm can send the complete large order directly to the exchange's trading system, without ever revealing the full size of the order to any market participant, including its own broker. Instead, only smaller lots are displayed to the market and automatically replaced when filled until the full order is finally executed. Says Thompson: 'Is this algorithmic trading? Some would say it was. To me, this is a fairly linear strategy that does not involve historical data and is pure DMA.'

\section{VIRTUAL TRADING}

Mario Muth, global head of electronic trading for listed derivatives, at Deutsche Bank, one of the earliest to enable fast DMA, says:

'DMA means there is no human intervention between the client and the exchange. It enables clients virtually to trade directly into the exchange. There are, however, complex risk checks in place and we ensure that the rules of the exchanges are adhered to. But ultimately it means that no one is manually intervening the client flow. Traditionally, when order flow was sent to traders, and for example there was an order larger than the available liquidity, the broker took these trades and worked them to manage the market impact. Today, algorithms can take care of the large orders and manage market impact.

'We have been doing DMA for listed derivatives for the last five years and the 
volume of business has significantly increased, with both existing clients and new customers. As these technologies become more cost effective they become more widely available to new users, so it is no longer just the large players using it for large orders. At the same time the increase in speed has allowed customers to be much closer to the market and react to price changes with an order amend or a new order.'

Deutsche Bank has written directly to the application programming interfaces of the derivatives exchanges. 'We have invested heavily in faster systems over the past three years and are continuing to closely examine trading speeds closely. Speed and stability are the most competitive issues as they make a difference between DMA systems.'

The other key element needed to enable DMA is liquidity. Algorithmic trading helps customers where liquidity is an issue. However, even algorithms need to find some liquidity and narrow bid-offer spreads. For this reason, algorithmic trading in derivatives still only really occurs in the futures market.

Deutsche Bank has developed specific algorithms in-house for high-volume equities clients, including money managers, pension funds and hedge funds, using algorithms every day. Muth adds, there is a another, group of clients who have a specific trading strategy and the bank gives them the 'ExchangeLink Complex Trading' system to help them to express those ideas into a systematically and trade them directly on the exchanges.

\section{HEDGE FUNDS WANT OWNERSHIP}

According to the providers of software for direct market access trading, buy-side firms are becoming more sophisticated, led by the increasing number of hedge funds that are starting up. Dr John Bates, Vice President Products, Progress Apama, says 'JP Morgan and Deutsche Bank use the Apama algorithmic trading platform for their institutional customers, but a growing number of hedge funds are coming direct to Progress as they want ownership of the algorithmic trading engines.'

According to Bates:

'Derivatives have almost leapfrogged equities in terms of what they can offer. The derivatives market is a little bit more forward thinking in terms of where the market is going. Equities were the only players in DMA and algorithmic trading for a long time. And when futures came along, they were behind, but they had a clean sheet of paper and could catch up very quickly. The buy-side realised that the way the market is going is DMA, and rather than rely upon the broker, they actually have the intellectual property on board to be able to carry out their own trading, by simply leasing the DMA capability from the broker and get access to the markets and the broker's order management systems.'

Bates believes hedge funds today are less likely to use black boxes because there is no advantage if everyone has access to the same algorithms. With black box trading, someone else has built the strategy, and the 
buy-side user cannot make any changes to it. The larger players, such as hedge fund Aspect Capital, which has recently bought the Apama platform to build their own algorithms, are now moving to 'white boxes', where they build and can tweak and tinker with the algorithms. Says Bates:

'Some smaller hedge funds, with fewer people, rent the algorithmic trading platforms from the broker. So they get introduced to algorithmic trading by the broker, and want to take control of the algorithms in-house. There is this area in the middle, of those who realise if they don't start building their own algorithms to differentiate themselves they will go out of business.'

\section{SINGLE PIPE TO MULTIPLE MARKETS}

Apama also offers the brokers adaptors to connect to different markets, such as GL for access to the world's exchanges, or the FX markets of EBS and Hotspot, so brokers can give their buy-side customers access to multiple markets through a single pipe. What many of the brokers are doing is buying Apama algorithmic trading platform and then building tailor-made strategies for the hedge funds, so that the black boxes they are using are in fact customised. The brokers are constantly creating new algorithms from what their customers want, and customising business strategies to match their customers' requirements.

Mark Palmer, Vice President, Event Stream Processing (ESP), Progress Apama, adds:
'A lot of the hedge funds are beginning to need the same technology as the brokers in order to gain competitive advantage. Direct market access and algorithmic trading are inextricably linked. Once you have access, combining it with algorithmic trading gives you the ability to apply greater intelligence to how you access the markets. If you don't innovate in this area, they won't survive, which is there has been has been such a rush to adopt new technology.'

Much of the focus on DMA is on speed - where a fraction of a second can make all the difference. Jon Steward, president of NYFIX Overseas in London, says that the electronic trading technology provider recently raised the ante in terms of speed by incorporating a tier one bank's order book into NYFIX's own network. Steward says: 'The fastest possible way of getting an order to the market is by writing a direct application programming interface (API) from the broker's to the exchange. We have gone one step further and taken a tier one broker's order management system and put it in the same room as the NYFIX network, which has a direct API connection to the CME.' This cuts down the split seconds sending the orders via a gateway, and instead the orders came directly from the NYFIX Order Book Management System. NYFIX provides global trade routing connectivity, straight through processing and execution services to worldwide equities, derivatives and fixed income financial markets. Through NYFIX, institutions have the ability to anonymously access new customisable technology 
designed to fit a wide range of trading strategies. These technologies include direct market access, the NYFIX Millennium traditional anonymous matching system and algorithmic trading models. According to Steward, the future trading of derivatives will be highly dependent on the sophistication of the DMA software. 'Software now manages an increasingly percentage of derivatives orders. This is moving so fast that it will be impossible to compete without sophisticated direct market access systems in place.'

\section{DMA EXPLOSION}

According to Paul MacGregor, director of technology partnerships at Euronext.liffe, there has been a real explosion in the number of member firms using direct market access since the exchange published an open application programming interface (API) a few years ago, McGregor says: 'There has been a distinct rise in the number of firms writing to our open API in the past year - from around 30 in the previous year to more than 100. While most are using DMA to connect their own internal trading desks to the exchange, some are providing onward connectivity for their customers. The trend is continuing, adds MacGregor, as the exchange continues to work with developers to support direct connections to the exchange across a broader coverage to include the cash and derivatives markets on a single platform.

Only a few years ago, the electronic trading landscape was one in which the major players were small software companies that were sometimes attached to equities brokers. These small software providers were running rings round their full-service competitors. Now, those same full-service brokers have acquired the some of the larger direct market access (DMA) providers and are busily folding them into their full-service environments. Within the last two years, Bank of America, Bank of New York and Citigroup acquired Direct Access Financial, Sonic Trading Management and Lava Trading, respectively. But, where will brokers take DMA next? So far, firms seem to be tentatively headed in all directions: international equities, foreign exchange and derivatives, seem to be in everyone's plans but it is becoming very clear that the gangbusters expansiveness of the booming '90s won't be making a comeback. 